\title{
EFECTO DEL ACABADO SOBRE LA CALIDAD DE LA CANAL DE TERNEROS Y TERNERAS ALIMENTADOS CON ENSILADOS
}

\author{
EFFECT OF FINISHING ON CARCASS QUALITY OF YOUNG BULLS AND HEIFFERS \\ FEEDINGSILAGE
}

\author{
Zea Salgueiro, J., M.D. Díaz Díaz y J.A. Carballo Santaolalla
}

Centro de Investigaciones Agrarias de Mabegondo. Apartado de Correos 10. 15008 A Coruña. España. jaime.zea.salgueiro@xunta.es; dolores.diaz.diaz@xunta.es

\author{
PaLABRAs CLAVE ADICIONALES \\ Ensilado de pradera. Ensilado de maíz.
}

\section{RESUMEN}

En 300 terneros y 300 terneras, alimentados a base de ensilados de pradera o maíz, se realizaron acabados con $405 \mathrm{~kg}$ de pienso o con pienso a voluntad, durante 45 o 90 días. Los animales se sacrificaron a los $400 \mathrm{~kg}$ de peso vivo los machos, y a $375 \mathrm{~kg}$ las hembras.

El rendimiento de la canal aumentó con el acabado. La mejora de la conformación que produce el acabado se manifiesta únicamente en los animales alimentados con ensilado de pradera (en las hembras sólo con acabados de 90 días y pienso a voluntad).

El engrasamiento también mejora con el acabado, tanto restringido como a voluntad. En el caso de las hembras únicamente en las alimentadas con ensilado de pradera. La grasa de riñonada aumentó con el acabado.

La composición tisular de la canal únicamente se vio afectada en el contenido en grasa, que aumentó con los acabados en el caso de los machos, y en el de las hembras únicamente en las alimentadas con ensilado de pradera.

Las mejoras que producen los acabados en la calidad de la canal son modestas y menores si los animales se alimentan con ensilado de maíz que si se hace con ensilado de pradera.

\section{SUMMARY}

Three hundred males and 300 females, fed on the basis of grass or maize silages, were finished with 4 or $5 \mathrm{~kg}$ of concentrate or with concentrate ad libitum during 45 or 90 days. The males were

Recibido: 15-2-07. Aceptado: 28-6-07.

\section{AdDitiOnAL KEYWORDS}

Grass silage. Maize silage.

slaughtered at $400 \mathrm{~kg}$ and females at $375 \mathrm{~kg}$ live weight.

The carcass dressing increased with finishing. The improvement of the conformation that the finishing produced, is only shown in animals fed with grass silage (in females it was only with finishing of 90 days and concentrate ad libitum).

Carcass fattening improves with finishing. In females it was only in the ones fed with grass silage. Kidney fat increased with finishing.

Carcass composition was only affected in the grass content, which increased with the finishings (in males), in females it was only in the ones fed with grass silage.

The improvements that produce the finishing in the carcass quality are modest and minor in the animals fed with maize silage than those fed with grass silage.

\section{INTRODUCCIÓN}

Se ha admitido que las condiciones que se les exigen a las canales, en especial en lo relativo a la conformación y al engrasamiento, son difíciles de alcanzar con animales de razas de maduración tardía alimentados a base de dietas forrajeras y sacrificados a pesos relativamente ligeros. Este es precisamente el caso de los animales de raza Rubia Gallega, Frisona o su cruce, que son los más abundantes en Galicia. De aquí, que se venga recomendando el acabado con 


\section{ZEASALGUEIRO, DÍAZDÍAZY CARBALLOSANTAOLALLA}

concentrado con el fin de mejorar, básicamente, la conformación y el engrasamiento de la canal.

Sin embargo, muchas veces el efecto del acabado se confunde con el que produce el aumento del peso de sacrificio. Así, en general, en un acabado que implica incremento del peso de sacrificio se produce la mejora de la conformación y, en conjunto, la calidad de la canal. Pero no hay que olvidar que estos mismos efectos se producen al incrementar el peso de sacrificio, de modo que el posible efecto del acabado y del peso de sacrificio se suelen confundir. Parece entonces oportuno realizar trabajos en los que los animales que son acabados con concentrados se sacrifiquen al mismo peso que los que no lo son, y poder discriminar así los efectos de los diferentes tratamientos.

\section{MATERIAL Y MÉTODOS}

Se utilizaron 300 terneros, 30 por tratamiento (10 de cada uno de los genotipos: Rubia Gallega, Frisona y su cruce) en un diseño factorial 2 × 5 (2 tipos de ensilado: pradera o maíz y 5 tratamientos: sin acabado, acabado de 45 días con pienso limitado $-5 \mathrm{~kg} /$ día si es con ensilado de pradera y $4 \mathrm{~kg}$ si es con ensilado de maíz-, acabado de 90 días con pienso limitado, acabado de 45 días con pienso a voluntad y acabado de 90 días con pienso a voluntad. Este diseño se repitió con hembras. Los machos se sacrificaron con $400 \mathrm{~kg}$ de peso y las hembras con $375 \mathrm{~kg}$. Dado que los pesos de sacrificio son fijos en cada sexo, el peso vivo con el que comenzaron los acabados fue distinto en cada uno de los tratamientos. Los sistemas de alimentación previos al comienzo de los tratamientos, iguales para machos y hembras, se esquematizan en la tabla I y las características de los ensilados en la tabla II.

Los piensos se constituyeron a base de harinas de cebada y soja y se formularon para que las ingestas resultasen con la proteína bruta que se indica en la tabla I. Los
Tabla I. Alimentación de los terneros. (Feeding systems).

\begin{tabular}{lcc}
\hline Ensilado de & maíz & pradera \\
\hline Hasta 270 kg de peso vivo & & \\
Ensilado & Ad libitum & Ad libitum \\
Pienso (kg/día) & 1,0 & 1,5 \\
Proteína bruta (\% dieta) & 14,0 & 14,0 \\
De 270 kg en adelante & & \\
Ensilado & Ad libitum Ad libitum \\
Pienso (kg/día) & 1,5 & 2,0 \\
Proteína bruta (\% dieta) & 12,0 & 12,0 \\
\hline
\end{tabular}

animales recibieron complementos vitamínico-minerales.

Las praderas de raigrás inglés, trébol blanco y algo de dactilo se ensilaron por corte directo (con 3 litros de ácido fórmico/ tonelada de materia verde) con una cose-

Tabla II. Características medias químicobromatológicas de los ensilados de maíz y pradera utilizados en los experimentos. (Average nutritive value of maize silage and grass silage).

\begin{tabular}{|c|c|c|}
\hline Ensilado & pradera & maíz \\
\hline Materia seca & 19,05 & 32,98 \\
\hline Materia orgánica & 90,28 & 96,75 \\
\hline Fibra ácido detergente & 36,37 & 23,71 \\
\hline Fibra neutro detergente & 55,02 & 41,85 \\
\hline Fibra bruta (celulosa) & 29,83 & 20,02 \\
\hline Cenizas & 7,72 & 3,25 \\
\hline Proteína bruta & 11,46 & 7,76 \\
\hline Digestibilidad materia orgánica & 64,71 & 68,97 \\
\hline Energía metabolizable (MJ/kg MS) & ) 9,39 & 10,60 \\
\hline $\mathrm{Ph}$ & 3,85 & 3,62 \\
\hline $\mathrm{pH}_{\mathrm{e}}$ & 4,15 & 4,64 \\
\hline \multicolumn{3}{|l|}{ Nitrógeno: } \\
\hline total & 1,83 & 1,21 \\
\hline amoniacal & 0,08 & 0,06 \\
\hline $\begin{array}{l}\mathrm{N}_{\mathrm{NH} 4} / \mathrm{N}_{\mathrm{T}} \\
\text { Ácidos: }\end{array}$ & 4,62 & 4,69 \\
\hline acético & 1,65 & 1,78 \\
\hline propiónico & 0,15 & 0,27 \\
\hline butírico & 0,26 & 0,01 \\
\hline láctico & 9,32 & 6,44 \\
\hline
\end{tabular}


Tabla III. Grados de conformación de la canal. (Carcass conformation grades).

\begin{tabular}{|c|c|c|c|c|c|c|c|c|c|c|c|c|c|c|}
\hline \multicolumn{3}{|c|}{$\mathrm{P}$} & \multicolumn{3}{|c|}{$\mathrm{O}$} & \multicolumn{3}{|c|}{$\mathrm{R}$} & \multicolumn{3}{|c|}{$\mathrm{U}$} & \multicolumn{3}{|c|}{$\mathrm{E}$} \\
\hline- & • & + & - & • & + & - & • & + & - & • & + & - & • & + \\
\hline 1 & 2 & 3 & 4 & 5 & 6 & 7 & 8 & 9 & 10 & 11 & 12 & 13 & 14 & 15 \\
\hline
\end{tabular}

chadora-picadora de doble corte. El maíz se ensiló directamente, en estado pastoso-vítreo, sin conservantes. La digestibilidad de la materia orgánica (DMO) se determinó in vitro por el método de Tilley y Terry (1963) modificado por Alexander(1969).

Previamente al comienzo de los periodos de control propiamente dichos, los terneros fueron desparasitados y se les sometió a un periodo de adaptación a las condiciones experimentales de 15 días. Los terneros se pesaron dos días consecutivos antes de servirles la ración y a la misma hora, al comienzo y al final de cada periodo experimental, con pesadas intermedias para mejor control. Los ensilados se suministraron para que siempre sobrase el 10-15\% de lo servido para asegurar que la ingestión fuese a voluntad.

Las canales se clasificaron según las normas de la UE (Reglamentos CEE n ${ }^{\circ} 1208 /$ 81 y 2930/81 y 2237/1991). La calificación de la conformación de las canales se determina atendiendo al desarrollo de los perfiles de las mismas, especialmente de la pierna, lomo y espalda. Las categorías adoptadas, dado que no existe la categoría $S$ que se corresponde con animales culones fueron: excelente (E), muy buena (U), buena (R), menos buena $(\mathrm{O})$ y mediocre $(\mathrm{P})$. La clasificación para el estado de engrasamiento se basó en la cantidad y distribución de la grasa de cobertura, con especial atención en el interior de la cavidad torácica, utilizándose la siguiente escala: no graso (1), poco cubierto
(2), cubierto (3), graso (4) y muy graso (5).

Para la clasificación de la conformación cada una de las cinco categorías fijadas fue precedida por un signo + o -, con lo que se estableció una escala de 15 puntos según el esquema indicado en la tabla III.

Para el engrasamiento se completaron las cinco categorías establecidas con un grado intermedio entre ellas, resultando así la escala con 9 puntos (tabla IV).

Para el despiece de la canal (Carballo et al., 2000) se utilizó la media canal izquierda, sin rabo, sin testículos y sin grasa renal. A las 48 horas post mórtem se procedió al cuarteo de la media canal al nivel de la $5^{\mathrm{a}} \mathrm{y}$ $6^{\mathrm{a}}$ costillas, dando como resultado el cuarto delantero y el trasero. Al retirar la falda en el cuarto trasero da lugar a la pistola. Se toma como peso de la canal fría el doble del sumatorio de todas las piezas, así como el hueso y la grasa obtenidas en el despiece. En el concepto carne se incluye la grasa intramuscular, en el de grasa todos los depósitos excepto el intramuscular y en el de hueso se incluyen los cartílagos, periostio y tejidos conectivos. Al retirarse la grasa renal se pesó y se determinó su porcentaje respecto a la canal (grasa de riñonada).

En la porción de lomo extraído a las 24 horas post mórtem y sobre papel de acetato con un rotulador de punta fina se dibujó el perímetro del músculo Longissimus thoracis de las costillas $6^{\mathrm{a}}$ y $10^{\mathrm{a}}$. Posteriormente se planimetraron las áreas dando los resultados en centímetros cuadrados. Además, se

Tabla IV. Grados de engrasamiento de la canal. (Carcass fattening grades).

\begin{tabular}{|c|c|c|c|c|c|c|c|c|}
\hline \multicolumn{2}{|c|}{ Magra } & \multicolumn{2}{|c|}{ Poco cubierta } & \multicolumn{2}{|c|}{ Cubierta } & \multicolumn{2}{|c|}{ Grasa } & \multirow{2}{*}{$\begin{array}{c}\text { Muy grasa } \\
\bullet\end{array}$} \\
\hline$\bullet$ & $+/-$ & 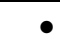 & $+/-$ & $\bullet$ & $+/-$ & $\bullet$ & $+/-$ & \\
\hline 1 & 2 & 3 & 4 & 5 & 6 & 7 & 8 & 9 \\
\hline
\end{tabular}




\section{ZEASALGUEIRO, DÍAZDÍAZY CARBALLOSANTAOLALLA}

midieron en el Longissimus thoracis las distancias A (diámetro mayor en sentido dorsoventral) y B (diámetro máximo, perpendicular al A), expresándolas en centímetros.

Los análisis estadísticos se realizan con el PROC ANOVA del paquete estadístico SAS (SAS Institute, 1985) siendo el modelo matemático:

$$
Y_{i j}=\eta+S_{i}+A_{j}+(S A)_{i j}+\varepsilon_{i j}
$$

donde:

Y son las variables en estudio, ganancias diarias de peso vivo y canal y cada una de las determinaciones que se hacen en la canal. $\eta$ es la media general.

$\mathrm{S}_{\mathrm{i}}$ es el efecto del tipo de ensilado.

A es el efecto del tipo de acabado.

$(\mathrm{SA})_{\mathrm{ij}}$ es la interacción de los factores $\mathrm{S}$ y $\mathrm{A}, \mathrm{y}$ $\varepsilon_{i j}$ es el error aleatorio $\mathrm{N}\left(0, \sigma^{2}\right)$.

\section{RESULTADOSYDISCUSIÓN}

De alguna manera las ganancias de peso siguieron a la cantidad de pienso consumido a lo largo de todo el ciclo y esto ocurre independientemente del ensilado consumido (tablas V y VI). Considerando las medias para los dos tipos de ensilados los que crecieron más rápidamente fueron los que tuvieron un acabado de 90 días con pienso

Tabla V. Machos: pesos inicial y final y al comienzo de los acabados ( $\mathrm{kg}$ ), así como ganancias de peso $(\mathrm{g} /$ día). (Males: Inicial and final liveweights $(\mathrm{kg})$ at the beginning of finishing and live weight gains $(g / d))$.

\begin{tabular}{|c|c|c|c|c|c|c|}
\hline \multirow{2}{*}{$\begin{array}{l}\text { Ensilado } \\
\text { Acabado } \\
\text { Pradera }\end{array}$} & \multirow[t]{2}{*}{$\begin{array}{l}\text { Peso } \\
\text { inicio }\end{array}$} & \multicolumn{2}{|c|}{$\begin{array}{l}\text { Peso vivo acabado } \\
\text { inicio al final }\end{array}$} & $\begin{array}{l}\text { Ganancia } \\
\text { preacabado }\end{array}$ & Ganancia de peso vivo en & $\begin{array}{l}\text { en } \\
\text { total }\end{array}$ \\
\hline & & & & & & \\
\hline Silo + 2 kg/día & 201,53 & - & 403,32 & 1044 & - & $1044^{\mathrm{a}}$ \\
\hline Acab 45 días 5 kg & 199,75 & $343,17^{a}$ & 403,17 & 1035 & $1354^{a}$ & $1113^{b}$ \\
\hline Acab 90 días 5 kg & 198,05 & $278,03^{b}$ & 402,18 & 1028 & $1374^{\mathrm{a}}$ & $1213^{c}$ \\
\hline Acab 45 días ad lib. & 202,42 & $328,85^{c}$ & 402,15 & 1009 & $1641^{\mathrm{b}}$ & $1178^{c}$ \\
\hline Acab 90 días ad lib. & 197,95 & $257,03^{d}$ & 401,85 & 1008 & $1609^{b}$ & $1381^{d}$ \\
\hline error típico & 3,539 & 3,906 & 3,707 & 15,387 & 28,125 & 16,615 \\
\hline nivel de significación & NS & $\star \star \star *$ & NS & NS & $* * *$ & $\star * *$ \\
\hline \multicolumn{7}{|l|}{ Maíz } \\
\hline Silo + 1,5 kg/día & 208,30 & - & 399,93 & 1260 & - & $1260^{\mathrm{a}}$ \\
\hline Acab 45 días 4 kg & 207,50 & $334,63^{a}$ & 401,08 & 1252 & $1500^{\mathrm{ab}}$ & $1329^{b}$ \\
\hline Acab 90 días 4 kg & 206,45 & $269,30^{b}$ & 402,55 & 1260 & $1480^{a}$ & $1400^{c}$ \\
\hline Acab 45 días ad lib. & 206,85 & $330,60^{a}$ & 400,70 & 1248 & $1558^{b}$ & $1345^{b}$ \\
\hline Acab 90 días ad lib. & 205,55 & $261,88^{b}$ & 399,65 & 1210 & $1530^{\mathrm{ab}}$ & $1425^{c}$ \\
\hline error típico & 3,575 & 3,392 & 3,752 & 18,429 & 23,974 & 16,925 \\
\hline nivel de significación & NS & $\star * \star$ & NS & NS & * & $* * *$ \\
\hline \multicolumn{7}{|l|}{ Medias } \\
\hline Silo + 1,75 kg/día & 204,92 & - & 401,62 & $1152^{\mathrm{a}}$ & - & $1152^{\mathrm{a}}$ \\
\hline Acab 45 días $4,5 \mathrm{~kg}$ & 203,63 & $338,90^{\mathrm{a}}$ & 402,13 & $1144^{\mathrm{a}}$ & $1427^{a}$ & $1221^{\mathrm{b}}$ \\
\hline Acab 90 días $4,5 \mathrm{~kg}$ & 202,25 & $273,67^{b}$ & 402,37 & $1144^{a}$ & $1427^{a}$ & $1306^{c}$ \\
\hline Acab 45 días ad lib. & 204,63 & $329,73^{c}$ & 401,43 & $1129^{a b}$ & $1599^{b}$ & $1262^{\mathrm{d}}$ \\
\hline Acab 90 días ad lib. & 201,75 & $259,46^{d}$ & 400,75 & $1109^{b}$ & $1570^{\mathrm{b}}$ & $1403^{e}$ \\
\hline error típico & 2,515 & 2,588 & 2,637 & 12,003 & 18,478 & 11,859 \\
\hline nivel de significación & NS & $* * *$ & NS & 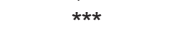 & $\star * *$ & $* * *$ \\
\hline
\end{tabular}

${ }^{* * *} p<0,001 ;{ }^{* *} p<0,01 ;{ }^{*} p<0,05 ;+p<0,1 ;$ N.S. no significativo.

En la misma columna cifras con distintas letras, son significativamente diferentes.

Archivos de zootecnia vol. 58, núm. 221, p. 14. 


\section{ACABADO Y CANAL DE TERNEROS Y TERNERAS ALIMENTADOS CON ENSILADOS}

a voluntad (1403 g/día los machos y $1179 \mathrm{~g} /$ día las hembras), seguidos de los que lo tuvieron de 90 días con pienso restringido (1306 y $1100 \mathrm{~g} /$ día, para machos y hembras) y por los que lo tuvieron de 45 días con pienso a voluntad (1262 y 1096 g/día, para machos y hembras) y de 45 días con pienso restringido (1221 g/día, los machos y $1051 \mathrm{~g} /$ día, las hembras). Los que menos crecieron fueron los que no recibieron ningún tipo de acabado y consumieron únicamente $1,75 \mathrm{~kg}$ de pienso al día como media (1152 y $1019 \mathrm{~g} /$ día, machos y hembras). En las hembras (tabla VI), entre las que consumieron $1,75 \mathrm{~kg}$ de pienso al día y las que tuvieron un aca- bado de 45 días con pienso limitado, no se observaron diferencias significativas; así como entre las que tuvieron acabados de 45 días con pienso a voluntad y las que lo tuvieron de 90 días con pienso restringido. Respuestas al concentrado de este tipo ya fueron observadas por Zea y Díaz (1996) para ensilado de pradera, y por Zea et al. (2001) con ensilado de maíz.

Las respuestas al concentrado, en ganancias diarias de peso vivo, fueron menores cuando la base de la alimentación fue el ensilado de maíz que cuando fue el ensilado de pradera. Con ensilado de pradera, en los periodos de acabado, las diferencias entre

Tabla VI. Hembras: pesos inicial y final y al comienzo de los acabados (kg), así como ganancias de peso ( $\mathrm{g} /$ día). (Females: Inicial and final liveweights $(\mathrm{kg})$ at the beginning of finishing and live weight gains $(\mathrm{g} / \mathrm{d}))$.

\begin{tabular}{|c|c|c|c|c|c|c|}
\hline $\begin{array}{l}\text { Ensilado } \\
\text { Acabado }\end{array}$ & $\begin{array}{l}\text { Peso } \\
\text { inicio }\end{array}$ & \multicolumn{2}{|c|}{ Peso vivo acabado } & \multicolumn{3}{|c|}{ Ganancia de peso vivo en } \\
\hline \multicolumn{7}{|l|}{ Pradera } \\
\hline Silo + 2 kg/día & 207,83 & - & 373,90 & 906 & - & $906^{a}$ \\
\hline Acab 45 días 5 kg & 203,06 & $322,59^{a}$ & 374,74 & 897 & $1159^{a}$ & $966^{b}$ \\
\hline Acab 90 días 5 kg & 203,79 & $273,53^{b}$ & 377,55 & 904 & $1156^{a}$ & $1044^{c}$ \\
\hline Acab 45 días ad lib. & 201,82 & $315,42^{\mathrm{a}}$ & 374,31 & 923 & $1309^{b}$ & $1028^{c}$ \\
\hline Acab 90 días ad lib. & 200,97 & $259,13^{b}$ & 375,44 & 911 & $1292^{\mathrm{b}}$ & $1135^{d}$ \\
\hline error típico & 3,222 & 3,721 & 2,888 & 11,744 & 18,173 & 19,321 \\
\hline nivel de significación & NS & $* * *$ & NS & NS & $\star \star * \star$ & $* * *$ \\
\hline \multicolumn{7}{|l|}{ Maíz } \\
\hline Silo + 1,5 kg/día. & 206,15 & - & 374,72 & 1163 & - & $1163^{a}$ \\
\hline Acab 45 días 4 kg & 206,08 & $321,93^{a}$ & 374,08 & 1152 & $1167^{a}$ & $1157^{\mathrm{a}}$ \\
\hline Acab 90 días 4 kg & 206,75 & $270,37^{b}$ & 376,17 & 1148 & $1175^{a}$ & $1167^{a}$ \\
\hline Acab 45 días ad lib. & 203,65 & $317,65^{a}$ & 373,92 & 1138 & $1250^{\mathrm{b}}$ & $1173^{a}$ \\
\hline Acab 90 días ad lib. & 203,97 & $259,98^{c}$ & 373,75 & 1124 & $1264^{b}$ & $1216^{b}$ \\
\hline error típico & 3,110 & 2,949 & 2,890 & 14,323 & 16,429 & 12,639 \\
\hline nivel de significación & NS & $\star * *$ & NS & NS & $* * *$ & $\star * *$ \\
\hline \multicolumn{7}{|l|}{ Medias } \\
\hline Silo + 1,75 kg/día & 209,24 & - & 374,27 & 1020 & - & $1019^{a}$ \\
\hline Acab 45 días $4,5 \mathrm{~kg}$ & 205,84 & $321,75^{a}$ & 374,55 & 1006 & $1171^{\mathrm{a}}$ & $1051^{\mathrm{a}}$ \\
\hline Acab 90 días $4,5 \mathrm{~kg}$ & 204,44 & $269,98^{b}$ & 375,64 & 1008 & $1174^{a}$ & $1100^{\mathrm{b}}$ \\
\hline Acab 45 días ad lib. & 202,15 & $315,01^{\mathrm{a}}$ & 372,95 & 1021 & $1288^{b}$ & $1096^{b}$ \\
\hline Acab 90 días ad lib. & 201,59 & $258,37^{c}$ & 374,16 & 1011 & $1286^{b}$ & $1179^{c}$ \\
\hline error típico & 3,211 & 3,042 & 3,651 & 8,225 & 11,736 & 15,406 \\
\hline nivel de significación & NS & $\star * *$ & NS & NS & $* * *$ & $\star * \star$ \\
\hline
\end{tabular}

${ }^{* * *} p<0,001 ;{ }^{* *} p<0,01 ;{ }^{*} p<0,05 ;+p<0,1 ;$ N.S. no significativo.

En la misma columna cifras con distintas letras, son significativamente diferentes. 


\section{ZEASALGUEIRO, DÍAZDÍAZY CARBALLOSANTAOLALLA}

los terneros (tabla $\mathbf{V}$ ) que recibieron $5 \mathrm{~kg}$ de concentrado o pienso a voluntad durante 45 días fueron de $287 \mathrm{~g} /$ día ( $<<0,001)$, y de 235 $\mathrm{g} /$ día $(\mathrm{p}<0,001)$ cuando el acabado fue de 90 días; mientras que con ensilado de maíz y 4 $\mathrm{kg}$ de pienso o con pienso a voluntad fue de $58 \mathrm{~g} /$ día (no significativo), con acabados de 45 días y de $50 \mathrm{~g} /$ día (NS) con acabados de 90 días.

En el caso de las hembras, las diferencias en las ganancias diarias de peso vivo como consecuencia de las respuestas al concen- trado se reducen en general, como puede deducirse de la tabla VI. Cuando la base de la alimentación fue el ensilado de pradera las diferencias en las ganancias de peso vivo, entre las terneras que en el acabado de 45 días consumieron $5 \mathrm{~kg}$ de pienso o pienso a voluntad, fue de $150 \mathrm{~g} /$ día $(\mathrm{p}<0,001)$, mientras que cuando la base de la alimentación fue el ensilado de maíz las diferencias en las ganancias de peso vivo fueron de $83 \mathrm{~g} /$ día $(p<0,001)$ y cuando el acabado fue de 90 días, estas cifras fueron $136(\mathrm{p}<0,001)$ y 89

Tabla VII. Machos: peso canal (kg), rendimiento (\%), clasificación canal, grasa de riñonada (\%) y \% de delantero, trasero y pistola en la canal. (Males: Carcass weight $(\mathrm{kg})$, dressing percentage, carcass clasification, kidney fat (\%) and forequarter, hindquarter and pistol in the carcass (\%)).

\begin{tabular}{|c|c|c|c|c|c|c|c|c|}
\hline $\begin{array}{l}\text { Ensilado } \\
\text { Acabado }\end{array}$ & $\begin{array}{l}\text { Peso } \\
\text { canal }\end{array}$ & $\begin{array}{l}\text { Rendimiento } \\
\qquad(\%)\end{array}$ & $\begin{array}{l}\text { Clasif } \\
\text { conf. }\end{array}$ & $\begin{array}{l}\text { canal }{ }^{1} \\
\text { engras. }\end{array}$ & $\begin{array}{l}\text { Grasa } \\
\text { riñón }\end{array}$ & \multicolumn{2}{|c|}{$\%$ en la canal de } & $\begin{array}{l}\text { de } \\
\text { pistola }\end{array}$ \\
\hline \multicolumn{9}{|l|}{ Prado } \\
\hline Silo + 2 kg/día & 209,05 & $51,82^{a}$ & $6,33^{a}$ & $3,80^{\mathrm{a}}$ & $1,17^{a}$ & 37,74 & 62,26 & 49,55 \\
\hline Acab 45 días 5 kg & 212,67 & $52,75^{\mathrm{b}}$ & $6,90^{\mathrm{a}}$ & $4,07^{\mathrm{ab}}$ & $1,26^{a}$ & 37,85 & 62,15 & 49,47 \\
\hline Acab 90 días 5 kg & 215,25 & $53,40^{c}$ & $7,23^{b}$ & $4,40^{b}$ & $1,33^{\mathrm{ab}}$ & 38,06 & 61,94 & 49,32 \\
\hline Acab 45 días ad lib. & 212,05 & $52,74^{b}$ & $7,17^{b}$ & $4,33^{b}$ & $1,45^{b}$ & 37,74 & 62,26 & 49,39 \\
\hline Acab 90 días ad lib. & 214,42 & $53,36^{c}$ & $7,43^{b}$ & $4,47^{b}$ & $1,47^{b}$ & 37,69 & 62,31 & 49,52 \\
\hline error típico & 2,358 & 0,336 & 0,248 & 0,163 & 0,057 & 0,192 & 0,192 & 0,214 \\
\hline nivel de significación & NS & $* * *$ & $* * *$ & ** & ** & NS & NS & NS \\
\hline \multicolumn{9}{|l|}{ Maíz } \\
\hline Silo + 1,5kg/día & 210,22 & $52,56^{a}$ & 7,03 & $3,67^{a}$ & $1,15^{a}$ & 37,50 & 62,50 & 49,61 \\
\hline Acab 45 días 4 kg & 211,95 & $52,82^{a}$ & 7,30 & $3,90^{\mathrm{ab}}$ & $1,18^{a}$ & 37,64 & 62,36 & 49,51 \\
\hline Acab 90 días 4 kg & 212,56 & $52,85^{a}$ & 7,30 & $3,97^{a b}$ & $1,26^{a}$ & 37,77 & 62,23 & 49,47 \\
\hline Acab 45 días ad lib. & 215,31 & $53,73^{b}$ & 7,40 & $4,17^{b}$ & $1,19^{a}$ & 37,80 & 62,20 & 49,34 \\
\hline Acab 90 días ad lib. & 215,64 & $53,96^{b}$ & 7,53 & $4,30^{b}$ & $1,48^{b}$ & 37,61 & 62,39 & 49,57 \\
\hline error típico & 2,371 & 0,327 & 0,270 & 0,160 & 0,048 & 0,168 & 0,169 & 0,172 \\
\hline nivel de significación & NS & ** & NS & * & $\star * \star$ & NS & NS & NS \\
\hline \multicolumn{9}{|l|}{ Medias } \\
\hline Silo + 1,75 kg/día & $209,63^{a}$ & $52,19^{a}$ & $6,68^{a}$ & $3,73^{a}$ & $1,16^{\mathrm{a}}$ & 37,62 & 62,38 & 49,58 \\
\hline Acab 45 días $4,5 \mathrm{~kg}$ & $212,31^{\mathrm{ab}}$ & $52,79^{a b}$ & $7,10^{\mathrm{ab}}$ & $3,98^{\mathrm{ab}}$ & $1,22^{\mathrm{ab}}$ & 37,74 & 62,26 & 49,49 \\
\hline Acab 90 días $4,5 \mathrm{~kg}$ & $213,91^{a b}$ & $53,17^{\mathrm{bc}}$ & $7,27^{\mathrm{b}}$ & $4,18^{b}$ & $1,29^{b}$ & 37,91 & 62,09 & 49,40 \\
\hline Acab 45 días ad lib. & $213,68^{a b}$ & $53,24^{\mathrm{cd}}$ & $7,28^{b}$ & $4,25^{\mathrm{b}}$ & $1,32^{b}$ & 37,77 & 62,23 & 49,37 \\
\hline Acab 90 días ad lib. & $215,03^{b}$ & $53,66^{d}$ & $7,48^{\mathrm{b}}$ & $4,38^{c}$ & $147^{c}$ & 37,65 & 62,35 & 4954 \\
\hline error típico & 1,672 & 0,234 & 0,183 & 0,114 & 0,037 & 0,128 & 0,129 & 0,137 \\
\hline nivel de significación & * & $\star * \star$ & $\star \star \star *$ & $* * *$ & $\star * \star$ & NS & NS & NS \\
\hline
\end{tabular}

${ }^{1}$ Conformación, de 1 a 15 . Engrasamiento, de 1 a 9.

${ }^{* * *} p<0,001 ;{ }^{* *} p<0,01 ;{ }^{*} p<0,05 ;+p<0,1$; N.S. no significativo.

En la misma columna cifras con distintas letras, son significativamente diferentes.

Archivos de zootecnia vol. 58, núm. 221, p. 16. 


\section{ACABADO Y CANAL DE TERNEROS Y TERNERAS ALIMENTADOS CON ENSILADOS}

$\mathrm{g} /$ día $(\mathrm{p}<0,001)$, respectivamente. Zea y Díaz (1990) ya habían observado que las respuestas al concentrado eran mucho mayores con ensilado de pradera que con ensilado de maíz, consecuencia del menor índice de sustitución del primero.

Como puede verse en las tablas VII y VIII el rendimiento a la canal aumenta cuando se realiza el acabado, tanto con concentrado limitado como con concentrado a voluntad. En los machos (tabla VII), cuando la base de la alimentación fue el ensilado de pradera, el rendimiento aumentó con la duración del acabado (igual en los dos niveles de concentrado): en 0,93 puntos porcentuales $(p<0,001)$ con acabado de 45 días y $5 \mathrm{~kg}$ de pienso y en 0,65 puntos $(\mathrm{p}<0,001)$ al pasar el acabado con $5 \mathrm{~kg}$ de pienso de 45 a 90 días. Si el acabado es con pienso a voluntad estas cifras en el mismo orden que antes son 0,92 puntos $(\mathrm{p}<0,001)$ y 0,62 $(\mathrm{p}<0,001)$ En el caso del ensilado de maíz, el rendimiento únicamente mejoró de modo significativo cuando el acabado se hizo con

Tabla VIII. Hembras: peso canal (kg), rendimiento (\%), clasificación canal, grasa de riñonada (\%) y \% de delantero, trasero y pistola en la canal. (Females: Carcass weight $(\mathrm{kg})$, dressing percentage, carcass clasification, kidney fat $(\%)$ and forequarter, hindquarter and pistol in the carcass $(\%)$ ).

\begin{tabular}{|c|c|c|c|c|c|c|c|c|}
\hline $\begin{array}{l}\text { Ensilado } \\
\text { Acabado }\end{array}$ & $\begin{array}{l}\text { Peso } \\
\text { canal }\end{array}$ & $\begin{array}{l}\text { Rendimiento } \\
\qquad(\%)\end{array}$ & $\begin{array}{l}\text { Clasif } \\
\text { conf. }\end{array}$ & $\begin{array}{l}\text { f. canal }{ }^{1} \\
\text { engras. }\end{array}$ & $\begin{array}{l}\text { Grasa } \\
\text { riñón }\end{array}$ & $\begin{array}{r}\% \text { en } \\
\text { delantero }\end{array}$ & $\%$ en la canal de & $\begin{array}{l}\text { de } \\
\text { pistola }\end{array}$ \\
\hline \multicolumn{9}{|l|}{ Pradera } \\
\hline Silo + 2 kg/día & 189,60 & $50,71^{a}$ & $5,93^{a}$ & $4,60^{\mathrm{a}}$ & $1,90^{\mathrm{a}}$ & 35,79 & 64,21 & 51,10 \\
\hline Acab 45 días 5 kg & 191,31 & $51,05^{b}$ & $6,37^{\mathrm{ab}}$ & $4,96^{b}$ & $2,20^{b}$ & 35,90 & 64,10 & 50,75 \\
\hline Acab 90 días 5 kg & 194,68 & $51,56^{c}$ & $6,47^{\mathrm{ab}}$ & $5,03^{b}$ & $2,31^{\mathrm{bc}}$ & 35,97 & 64,03 & 50,63 \\
\hline Acab 45 días ad lib. & 193,07 & $51,58^{c}$ & $6,50^{\mathrm{ab}}$ & $5,10^{\mathrm{b}}$ & $2,46^{c}$ & 35,89 & 64,11 & 50,81 \\
\hline Acab 90 días ad lib. & 194,18 & $51,73^{c}$ & $6,57^{\mathrm{b}}$ & $5.20^{\mathrm{b}}$ & $2,49^{c}$ & 35,88 & 64,12 & 50,74 \\
\hline error típico & 1,831 & 0,259 & 0,218 & 0,127 & 0,087 & 0,144 & 0,144 & 0,180 \\
\hline nivel de significación & + & $* *$ & * & ** & $* * *$ & NS & NS & + \\
\hline \multicolumn{9}{|l|}{ Maíz } \\
\hline Silo + 1,5 kg/día & $190,42^{a}$ & $50,83^{a}$ & 6,50 & 4,83 & $1,97^{a}$ & 35,77 & 64,17 & 50,67 \\
\hline Acab 45 días 4 kg & $192,24^{\mathrm{ab}}$ & $51,37^{b}$ & 6,63 & 4,97 & $2,18^{b}$ & 35,78 & 64,22 & 50,71 \\
\hline Acab 90 días 4 kg & $193,24^{\mathrm{ab}}$ & $51,71^{b}$ & 6,80 & 4,97 & $2,23^{b c}$ & 35,52 & 64,48 & 50,65 \\
\hline Acab 45 días ad lib. & $193,04^{\mathrm{ab}}$ & $51,96^{b}$ & 6,70 & 4,97 & $2,37^{b c}$ & 35,72 & 64,28 & 50,74 \\
\hline Acab 90 días ad lib. & $195,29^{b}$ & $52,37^{b}$ & 6,77 & 5,03 & $2,43^{c}$ & 35,83 & 64,17 & 50,69 \\
\hline error típico & 1,665 & 0.288 & 0,243 & 0.146 & 0,071 & 0,177 & 0,177 & 0,192 \\
\hline nivel de significación & * & $\star \star \star *$ & NS & NS & $* \star *$ & NS & NS & NS \\
\hline \multicolumn{9}{|l|}{ Medias } \\
\hline Silo + 1,75 kg/día & $190,01^{a}$ & $50,77^{a}$ & 6,22 & $4,72^{\mathrm{a}}$ & $1,94^{a}$ & 35,78 & 64,25 & 50,88 \\
\hline Acab 45 días $4,5 \mathrm{~kg}$ & $191,78^{b}$ & $51,21^{\mathrm{ab}}$ & 6,50 & $4,97^{\mathrm{ab}}$ & $2,19^{b c}$ & 35,84 & 64,16 & 50,73 \\
\hline Acab 90 días $4,5 \mathrm{~kg}$ & $193,96^{\mathrm{ab}}$ & $51,64^{\mathrm{bc}}$ & 6,63 & $5,00^{\mathrm{b}}$ & $2,27^{\mathrm{cd}}$ & 35,75 & 64,25 & 50,64 \\
\hline Acab 45 días ad lib. & $193,05^{\mathrm{ab}}$ & $51,77^{c}$ & 6,60 & $5,03^{b}$ & $2,41^{\text {de }}$ & 35,81 & 64,19 & 50,77 \\
\hline Acab 90 días ad lib. & $194,74^{b}$ & $52,05^{c}$ & 6,67 & $5,12^{\mathrm{b}}$ & $2,46^{e}$ & 35,85 & 64,15 & 50,72 \\
\hline error típico & 1,237 & 0,194 & 0,163 & 0,097 & 0,056 & 0,114 & 0,114 & 0,131 \\
\hline nivel de significación & $* *$ & $\star * *$ & NS & ** & $\star \star *$ & NS & NS & NS \\
\hline
\end{tabular}

${ }^{1}$ Conformación, de 1 a 15 . Engrasamiento, de 1 a 9.

${ }^{* * *} p<0,001 ;{ }^{* *} p<0,01 ;{ }^{*} p<0,05 ;+p<0,1 ;$ N.S. no significativo.

En la misma columna cifras con distintas letras, son significativamente diferentes. 


\section{ZEASALGUEIRO, DÍAZDÍAZY CARBALLOSANTAOLALLA}

pienso a voluntad, independientemente de la duración del acabado: en 1,17 puntos $(\mathrm{p}<0,01)$ con acabado de 45 días y en 0,23 puntos (NS) al pasar el acabado de 45 a 90 días.

En las hembras (tabla VIII) alimentadas a base de ensilado de pradera el rendimiento también mejoró con la duración del acabado con pienso limitado: en 0,34 puntos $(\mathrm{p}<0,01)$ y en 0,51 puntos $(\mathrm{p}<0,01)$ al hacerse acabado durante 45 días y al pasar éste de 45 a 90 días. Con pienso a voluntad la mejora no aumentó con la duración del acabado. En las terneras alimentadas con ensilado de maíz los acabados, sean con pienso limitado o con pienso a voluntad, no produjeron mejoras significativas en el rendimiento canal más allá de los 45 días: en 0,54 puntos $(\mathrm{p}<0,01)$ y 1,13 puntos $(\mathrm{p}<0,001)$ con acabados de 45 días y ensilados de pradera o maíz, respectivamente; y en 0,36 y 0,41 puntos, diferencias no significativas, al pasar la duración de los acabados de 45 a 90 días, según sean terneras alimentadas a base de ensilados de pradera o maíz.

En líneas generales, se puede decir que los rendimientos mejoraron de acuerdo con la calidad energética de la dieta recibida, lo

Tabla IX. Machos: composición de la canal(\%) y áreas de Longissimus thoraci al nivel de la $6^{a}$ y $10^{a}$ costillas $\left(\mathrm{cm}^{2}\right)$. (Males: Carcass composition (\%) and Longissimus thoraci area of the $6^{\text {th }}$ and $10^{\text {th }}$ ribs $\left.\left(\mathrm{cm}^{2}\right)\right)$.

\begin{tabular}{|c|c|c|c|c|c|c|c|c|c|}
\hline \multirow{2}{*}{$\begin{array}{l}\text { Ensilado } \\
\text { Acabado }\end{array}$} & \multicolumn{3}{|c|}{$\%$ en la canal de } & \multicolumn{2}{|c|}{ Area (costilla) } & \multicolumn{4}{|c|}{ \% de carne en la canal } \\
\hline & carne & hueso & grasa & $10^{a}$ & $6^{a}$ & extra & $1^{\mathrm{a}}$ & $2^{\mathrm{a}}$ & $3^{a}$ \\
\hline \multicolumn{10}{|l|}{ Pradera } \\
\hline Silo + 2 kg/día & 74,40 & 20,69 & $4,90^{\mathrm{a}}$ & 64,88 & 29,82 & 10,39 & 38,99 & 6,85 & 17,69 \\
\hline Acab 45 días $5 \mathrm{~kg}$ & 74,27 & 20,83 & $4,88^{a}$ & 65,47 & 30,15 & 10,38 & 39,19 & 6,89 & 17,64 \\
\hline Acab 90 días $5 \mathrm{~kg}$ & 74,09 & 20,58 & $5,32^{b}$ & 65,69 & 30,55 & 10,41 & 39,06 & 6,81 & 17,59 \\
\hline Acab 45 días ad lib. & 73,98 & 20,83 & $5,18^{\mathrm{ab}}$ & 65,28 & 30,38 & 10,30 & 39,00 & 6,82 & 17,69 \\
\hline Acab 90 días ad lib. & 73,89 & 20,69 & $5,42^{b}$ & 65,79 & 30,29 & 10,33 & 38,84 & 6.80 & 17,77 \\
\hline error típico & 0,267 & 0,293 & 0,149 & 1,417 & 0,877 & 0,192 & 0,238 & 0,086 & 0,180 \\
\hline nivel de significación & + & + & $\star *$ & NS & + & + & NS & + & NS \\
\hline \multicolumn{10}{|l|}{ Maíz } \\
\hline Silo + 1,5 kg/día & 74,93 & 20,27 & $4,80^{\mathrm{a}}$ & 65,01 & 30,61 & 10,96 & 39,60 & 6,52 & 17,63 \\
\hline Acab 45 días $4 \mathrm{~kg}$ & 75,14 & 19,94 & $4,91^{\mathrm{ab}}$ & 65,73 & 31,09 & 11,01 & 39,79 & 6,49 & 17,69 \\
\hline Acab 90 días 4 kg & 75,10 & 19,80 & $5,10^{b}$ & 65,78 & 30,96 & 11,05 & 39,70 & 6,39 & 17,80 \\
\hline Acab 45 días ad lib. & 74,94 & 20,10 & $5,07^{b}$ & 66,34 & 31,39 & 10,86 & 39,48 & 6,57 & 17,77 \\
\hline Acab 90 días ad lib. & 74,53 & 20,16 & $5,30^{b}$ & 66,77 & 31,60 & 10,81 & 39,32 & 6,53 & 17,73 \\
\hline error típico & 0,260 & 0,233 & 0,140 & 1,283 & 0,602 & 0,179 & 0,271 & 0,099 & 0,184 \\
\hline nivel de significación & NS & NS & 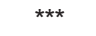 & NS & NS & NS & NS & NS & NS \\
\hline \multicolumn{10}{|l|}{ Medias } \\
\hline Silo + 1,75 kg/día & 74,67 & 20,48 & $4,85^{\mathrm{a}}$ & 64,95 & 30,21 & 10,68 & 39,30 & 6,68 & 17,66 \\
\hline Acab 45 días $4,5 \mathrm{~kg}$ & 74,71 & 20,38 & $4,90^{\mathrm{ab}}$ & 65,60 & 30,62 & 10,70 & 39,49 & 6,69 & 17,67 \\
\hline Acab 90 días $4,5 \mathrm{~kg}$ & 74,60 & 20,19 & $5,21^{c}$ & 65,73 & 30,75 & 10,73 & 39,38 & 6,60 & 17,69 \\
\hline Acab 45 días ad lib. & 74,46 & 20,46 & $5,12^{\mathrm{bc}}$ & 65,81 & 30,89 & 10,58 & 39,24 & 6,69 & 17,73 \\
\hline Acab 90 días ad lib. & 74,21 & 20,43 & $5,36^{c}$ & 66,28 & 30,95 & 10,57 & 39,08 & 6,67 & 17,75 \\
\hline error típico & 0,186 & 0,161 & 0,102 & 0,956 & 0,472 & 0,166 & 0,180 & 0,059 & 0,129 \\
\hline nivel de significación & NS & NS & $\star \star \star *$ & NS & NS & NS & NS & NS & NS \\
\hline
\end{tabular}

${ }^{* * *} p<0,001 ;{ }^{* *} p<0,01 ;{ }^{*} p<0,05 ;+p<0,1 ;$ N.S. no significativo.

En la misma columna cifras con distintas letras, son significativamente diferentes.

Archivos de zootecnia vol. 58, núm. 221, p. 18. 


\section{ACABADO Y CANAL DE TERNEROS Y TERNERAS ALIMENTADOS CON ENSILADOS}

que era de esperar ya que el factor más importante que afecta al rendimiento es el contenido intestinal, y éste depende, en gran medida, del tipo de dieta. Cuanto más alta sea la calidad de la dieta más bajo será el contenido digestivo y viceversa (Zea y Díaz, 1990). Keane (1981) observó que aumentando la cantidad de concentrados en dietas a base de forrajes disminuía el contenido intestinal y aumentaba el rendimiento canal de terneros.

En su conjunto, el efecto del acabado en la mejora de la conformación únicamente se produce en las canales de los machos (tabla
VII). La mejora de la conformación de la canal es en los machos de 0,59 puntos $(\mathrm{p}<0,001)$, cuando el acabado de 90 días se hace con concentrado limitado y de 0,80 $(\mathrm{p}<0,001)$ cuando se hace con concentrado a voluntad (con acabados de 45 días no se producen mejoras significativas de la conformación de las canales). En las hembras ni los acabados de 45 días ni los de 90 días produjeron mejoras significativas en la conformación de las canales (tabla VIII).

Sin embargo, si se estudian los efectos de los acabados en la conformación de las canales, según los animales sean alimenta-

Tabla X. Hembras: composición de la canal(\%) y áreas de Longissimus thoraci al nivel de la $6^{a}$ y $10^{a}$ costillas $\left(\mathrm{cm}^{2}\right)$. (Females: Carcass composition (\%) and Longissimus thoraci area of the $6^{\text {th }}$ and $10^{\text {th }}$ ribs $\left.\left(\mathrm{cm}^{2}\right)\right)$.

\begin{tabular}{|c|c|c|c|c|c|c|c|c|c|}
\hline \multirow{2}{*}{$\begin{array}{l}\text { Ensilado } \\
\text { Acabado }\end{array}$} & \multicolumn{3}{|c|}{$\%$ en la canal de } & \multicolumn{2}{|c|}{ Area (costilla) } & \multicolumn{4}{|c|}{$\%$ de carne en la canal } \\
\hline & carne & hueso & grasa & $10^{a}$ & $6^{a}$ & extra & $1^{a}$ & $2^{\mathrm{a}}$ & $3^{a}$ \\
\hline \multicolumn{10}{|l|}{ Pradera } \\
\hline Silo + 2 kg/día & 73,30 & 20,23 & $6,26^{a}$ & 58,92 & 28,52 & 10,76 & 38,07 & 6,56 & 17,84 \\
\hline Acab 45 días 5 kg & 73,52 & 20,07 & $6,41^{\mathrm{ab}}$ & 59,07 & 29,04 & 10,80 & 38,03 & 6,62 & 17,93 \\
\hline Acab 90 días 5 kg & 73,33 & 19,89 & $6,78^{\mathrm{ab}}$ & 59,09 & 29,28 & 10,83 & 37,85 & 6,60 & 17,92 \\
\hline Acab 45 días ad lib. & 73,19 & 20,16 & $6,64^{\mathrm{ab}}$ & 59,21 & 28,84 & 10,74 & 37,79 & 6,48 & 17,99 \\
\hline Acab 90 días ad lib. & 72,86 & 20,22 & $6,92^{b}$ & 58,91 & 28,54 & 10,74 & 37,74 & 6,50 & 17,62 \\
\hline error típico & 0,280 & 0,173 & 0,227 & 1,114 & 0,610 & 0,152 & 0,260 & 0,074 & 0,157 \\
\hline nivel de significación & NS & NS & * & NS & NS & NS & NS & NS & NS \\
\hline \multicolumn{10}{|l|}{ Maíz } \\
\hline Silo + 1,5 kg/día & 73,27 & 20,06 & 6,67 & 57,56 & 28,06 & 10,83 & 37,83 & 6,37 & 17,63 \\
\hline Acab 45 días 4 kg & 73,35 & 19,79 & 6,86 & 57,68 & 28,34 & 10,94 & 37,89 & 6,36 & 17,62 \\
\hline Acab 90 días 4 kg & 73,11 & 19,89 & 6,97 & 58,32 & 28,14 & 10,89 & 37,80 & 6,31 & 17,70 \\
\hline Acab 45 días ad lib. & 73,31 & 19,75 & 6,95 & 58,02 & 28,10 & 10,92 & 38,12 & 6,31 & 17,62 \\
\hline Acab 90 días ad lib. & 73,24 & 19,68 & 7,07 & 57,78 & 28,45 & 10,90 & 37,99 & 6,34 & 17,69 \\
\hline error típico & 0,284 & 0,194 & 0,211 & 1,167 & 0,793 & 0,124 & 0,263 & 0,067 & 0,158 \\
\hline nivel de significación & NS & NS & NS & NS & NS & NS & NS & NS & NS \\
\hline \multicolumn{10}{|l|}{ Medias } \\
\hline Silo + 1,75 kg/día & 73,39 & 20,14 & 6,47 & 58,24 & 28,29 & 10,83 & 37,95 & 6,47 & 17,73 \\
\hline Acab 45 días $4,5 \mathrm{~kg}$ & 73,44 & 19,93 & 6,63 & 58,38 & 28,69 & 10,94 & 37,96 & 6,49 & 17,77 \\
\hline Acab 90 días $4,5 \mathrm{~kg}$ & 73,22 & 19,87 & 6,88 & 58,71 & 28,71 & 10,89 & 37,82 & 6,45 & 17,81 \\
\hline Acab 45 días ad lib. & 73,25 & 19,96 & 6,80 & 58,62 & 28,47 & 10,92 & 37,96 & 6,39 & 17,80 \\
\hline Acab 90 días ad lib. & 73,05 & 19,95 & 7,00 & 58,35 & 28,49 & 10,89 & 37,87 & 6,42 & 17,73 \\
\hline error típico & 0,199 & 0,130 & 0,155 & 0,807 & 0,388 & 0,113 & 0,185 & 0,050 & 0,111 \\
\hline nivel de significación & NS & NS & NS & NS & NS & NS & NS & NS & NS \\
\hline
\end{tabular}

${ }^{* * *} p<0,001 ;{ }^{* *} p<0,01 ;{ }^{*} p<0,05 ;+p<0,1 ;$ N.S. no significativo.

En la misma columna cifras con distintas letras, son significativamente diferentes. 


\section{ZEASALGUEIRO, DÍAZDÍAZY CARBALLO SANTAOLALLA}

dos con uno u otro ensilado, se observa que las mejoras se producen únicamente en las canales de los alimentados con ensilado de pradera y no en los que consumieron ensilado de maíz. En los terneros (tabla VII), cuando el acabado se hace con $5 \mathrm{~kg}$ de pienso por cabeza y día, hay que llegar a 90 días para que la mejora sea significativa; con pienso a voluntad la mejora se produce a los 45 días, pero no aumenta al alargar el acabado. En las terneras (tabla VIII) las mejoras significativas sólo se producen con acabados de 90 días y pienso a voluntad (aunque la conformación de las canales obtenidas con acabados de 45 o 90 días no resultaron significativamente diferentes).

Los acabados mejoraron el engrasamiento canal, tanto en los machos como en las hembras. En los machos (tabla VII) en 0,45 puntos $(\mathrm{p}<0,001)$ y 0,65 puntos $(\mathrm{p}<0,001)$ cuando los acabados de 90 días se realizaron con pienso limitado o con pienso a voluntad, y en las hembras (tabla VIII) en $0,28(\mathrm{p}<0,01)$ y $0,40(\mathrm{p}<0,01)($ también con acabados de 90 días y con pienso limitado o a voluntad). Cuando el acabado se hace con pienso limitado la mejora del engrasamiento es significativa, únicamente cuando el acabado dura 90 días y no 45 días (en machos y en hembras). En el caso de las hembras la mejora del engrasamiento únicamente se produjo en las canales de las terneras alimentadas con ensilado de pradera. Esto se debería a que las canales de las hembras alimentadas con ensilado de maíz estarían más engrasadas al ser la alimentación más energética. La grasa de riñonada sigue, en líneas generales, la misma pauta que el engrasamiento, aumenta con los acabados; en este caso, incluso, en las hembras alimentadas con ensilado de maíz.

Andersen et al. (1984) ya habían observado que el nivel energético de la dieta influye directamente en la conformación y en el engrasamiento de las canales. Estos resultados parecen confirmar otros ya existentes (Cabrero, 1991), que indican que con el incremento del nivel energético de la ración aumenta el engrasamiento, y con ello mejora la conformación, como de hecho pudieron comprobarlo Zea et al. (1997) o Pena (2000), siendo estos cambios más drásticos en las hembras que en los machos (Varela, 2002). Por otra parte, el que la intensidad de las mejoras en la conformación y el engrasamiento sea mayor en los animales alimentados con el ensilado de pradera se debe a que el incremento de la riqueza energética de la dieta de acabado con el aumento de pienso es mayor con este ensilado. No hay que olvidar que el valor energético del ensilado de pradera era de 9,39 MJ EM/kg MS; mientras que el de maíz era de 10,60 MJ de EM/ kg de MS (tabla II).

Ni la duración del acabado ni la cantidad de pienso afectaron a los porcentajes de delantero, trasero o pistola, lo que fue independiente del tipo de forraje consumido y del sexo (tablas VII y VIII).

De la observación de la tabla IX se deduce que los acabados afectaron significativamente a la composición tisular de las canales de los machos, en las que la grasa aumentó. En el caso de ensilado de pradera con acabado con $5 \mathrm{~kg}$ de pienso a partir de los 90 días y con el acabado con pienso a voluntad a partir de los 45 días, y en los alimentados con ensilado de maíz la grasa aumentó de forma significativa, sólo si el acabado se hace con pienso a voluntad y se produce a partir de los 45 días (tabla IX). En las hembras (tabla $\mathbf{X}$ ), si se consideran conjuntamente los dos forrajes, no se aprecian efectos de los acabados en la composición tisular de la canal, pero sin embargo, el acabado con pienso a voluntad en las terneras alimentadas a base de ensilado de pradera sí aumentó el contenido grasa de las canales (tabla X). Los acabados, en ningún caso, afectaron significativamente a los contenidos de carne o hueso en las canales.

La proporción de carne de distintas calidades comerciales en la canal o las áreas del L. thoracis, determinadas al nivel de la $6^{\mathrm{a}}$ o $10^{\mathrm{a}}$ costillas, no fueron modificadas por los acabados en ninguna de sus formas, ni 
en los machos ni en las hembras, ya sean alimentadas a base de ensilado de pradera o de maíz (tablas IX y X).

Los escasos efectos observados en la composición de las canales parecen contradecir lo expuesto por Waldman et al. (1971) o por Keane y Drennan (1980) de que un aumento de la ingestión energética lleva a una disminución de los porcentajes de carne y hueso y al aumento del de grasa. No obstante, la cuantificación de estos cambios ha sido con frecuencia motivo de controversia, fundamentalmente en aquellos casos en que las variaciones de los tejidos han sido acompañadas de diferencias en peso de la canal (Cabrero, 1991). Los mismos Keane y Drennan (1980) indican que estos efectos se acentúan con el aumento del peso canal, y no hay que olvidar que nuestras canales resultaron bastante ligeras. Por otra parte, el efecto del nivel energético de la dieta en la canal es mucho más evidente en las razas más precoces (Geay et al., 1976) y no es tan evidente cuando se consideran razas menos precoces y de mayor formato (Cabrero, 1991). En este caso, la respuesta al nivel energético resulta variable, dependiendo del tipo genético del animal (Geay y Beranger, 1969; Geay et al., 1976). Para una misma edad, en las canales de razas más precoces, el aumento de la energía incrementa considerablemente los depósitos adiposos y disminuye el de músculo y hueso. En animales de razas más tardías que es nuestro caso, los tejidos evolucionan todos de forma similar y no se

\section{BIBLIOGRAFÍA}

Alexander, R.L. 1969. The establishment of a laboratory procedure for the in vitro determination of digestibility. The West Scotland College. Res. Bull., n 42, 52 p.

Andersen, H.R., K.C. Ingvarsten and H. Klastrup. 1984. Influence of energy level, weight at slaughter and castration on carcass quality in cattle. Livest. Prod. Sci., 11: 571-586.

Cabrero, M. 1991. Estructura y composición de la modifica, sustancialmente la composición (Geay et al., 1976). En este sentido cabe explicar nuestros resultados, que coincidirían con los obtenidos por Zea (1978) para la composición de las canales de terneros sometidos a dietas con diferente composición energética de la raza Rubia Gallega y sacrificados a $400 \mathrm{~kg}$ de peso vivo.

\section{CONCLUSIONES}

Las mejoras que producen los acabados en la calidad de la canal son modestas y menores si los animales se alimentan con ensilado de maíz que si se hace con ensilado de pradera. Los acabados producen la mejora del rendimiento y del engrasamiento. $\mathrm{La}$ conformación de las canales mejoran únicamente si los animales se alimentan con ensilado de pradera, pero no si se hace con ensilado de maíz.

\section{AGRADECIMIENTOS}

A D. Eduardo Jiménez Domínguez y a D. José Tasende Fraga, por el cuidado y la atención del ganado experimental y al INIA por la financiación del Proyecto: XM-99003 (Mejora de la calidad de la canal y la carne de vacuno joven alimentado a base de forrajes ensilados) y a la Secretaría Xeral de I+D de la Xunta de Galicia que financió el Proyecto: PGIDT02RAG50301PR(Efecto de la raza, sexo, peso de sacrificio y dieta en el perfil de los ácidos grasos de la carne de vacuno joven), de donde proceden los datos aquí expuestos.

canal como determinantes de su calidad. Bovis, 38: 9-37.

Carballo, J.A., L. Monserrat y L. Sánchez. 2000. Composición de la canal bovina. En: Metodología para el estudio de la calidad de la canal y de la carne en rumiantes, Coed.: V. Cañeque; C Sañudo. Monografías INIA. Serie Ganadera ${ }^{\circ}$ 1 Madrid. p. 173-179.

CEE. 1981. Reglamento nº 1208/1981 de La Comi-

Archivos de zootecnia vol. 58, núm. 221, p. 21. 


\section{ZEASALGUEIRO, DÍAZDÍAZY CARBALLO SANTAOLALLA}

sión Europea de 28 de abril de 1981

CEE. 1981. Reglamento n² 2930/1981 de La Comisión Europea de 21 de octubre de 1981.

CEE. 1991. Reglamento n²237/1991 de La Comisión Europea de 27 de julio de 1991.

Geay, Y. et C. Beranger. 1969. Influence de la proportion de céréales dans a ration sur l'etat d'engrasseiment des carcasse de taurillons de 15 mois. Ann. Zootech., 18: 79-91.

Geay, Y., J. Robelin et C. Berenger. 1976. Influence du niveau alimentaire sur le gain de poid vif et la composition de la carcasse de taurillons de differentes races. Ann. Zootech., 25: 287-302.

Keane, G. 1981. Carcass growth and compositon. In: Cattle Production Seminar. Grange Research Station. An Foras Taluntais. Dublin. Paper 15.

Keane, G. and M.J. Drennan. 1980. Effects of diet type and feeding levels on performance, carcass composition and efficiency of Friesian steer serially slaughtred. Ir. J. Agric. Res., 19: 53-67.

Pena, M.J. 2000. Utilización de ensilados de leguminosas en mezcla con el de maíz para la producción de carne de vacuno joven. Tesis Doctoral. Universidad de Santiago de Compostela.

SAS Institute. 1985. SAS User's guide: Statistic basic. version $5^{\text {a }}$ ed. SAS Institute Inc., Cary, N.C.

Tilley, J.A.M. and R.A. Terry. 1963. A two-stage technique for the in vitro digestion of forage crops. J. Brit. Grassl. Soc., 18: 104-111.

Varela, A. 2002. Estudio de las variables que afectan a la producción del tipo "Cebón". Tesis Doctoral. Universidad de Santiago de Compostela.

Waldman, R.C., W.J. Tyler and W.H. Brungardt. 1971. Changes in the carcass composition of Holstein steer associated with ration energy levels and growth. J. Anim. Sci., 32: 611-624.

Zea, J. 1978. Utilización de forrajes en la alimentación intensiva para la producción de añojos de la raza Rubia Gallega. Colección Tesis Doctorales INIA n 10. Madrid.

Zea, J. y M.D. Díaz. 1990. Producción de carne con pastos y forrajes. Mundi Prensa. $389 \mathrm{p}$.

Zea, J. y M.D. Díaz. 1996. Utilización de pastos y ensilados en la producción de carne de vacuno. Pastos, 26: 129-173.

Zea, J., M.D. Díaz y M.J. Pena. 1997. Efecto de la suplementación energética y proteica en la canal y la carne de terneros alimentados a base de ensilado de pradera. Actas de la XXXVI Reunión Científica de la SEEP, 489-494. SeviIla.

Zea, J., J.A. Carballo y M.D. Díaz. 2001. Efecto del sexo y de la suplementación energética en raciones isoproteicas a base de ensilado de maíz para el crecimiento y engorde de terneros Rubio Gallegos, I. Parametros productivos y características de las canales. Rev. Portuguesa Zootecn., 8: 87-97.

Archivos de zootecnia vol. 58, núm. 221, p. 22. 